\title{
Research on the remineralization of partially decalcified enamel surfaces using extracted tooth with calcifying solution
}

\author{
Maki Minami, Shigeru Watanabe, Akira Suzuki and Chenghua Pai \\ Department of Human Development \& Fostering, Division of Pediatric Dentistry, \\ School of Dentistry, Meikai University \\ 1-1 Keyakidai, Sakado, Saitama 350-0283, JAPAN
}

\begin{abstract}
OBJECTIVE: The recovery of tooth enamel after acid etching was examined in vitro using enamel powder in order to maximize the contact surface area. METHODS: The weight of the powder decreased due to acid etching and then increased when the powder was exposed to a calcifying solution. The increase in weight was due to the formation of needle like crystals. According to thermal gravimetric analysis, the newly formed enamel layer was very stable, and was suggested to be apatite by X-ray diffraction and FT-Raman analysis. There was no distinct difference between the newly formed layer and the original tooth in terms of composite $\mathrm{X}$-ray image $(\mathrm{CaK} \alpha$ and $\mathrm{PK} \alpha$ ). The new layer appeared to be a crystallized extension of the tooth enamel, as determined from SEM observations. However, the outer layer of tooth enamel was damaged by acid etching, and the crystallization had deteriorated 48 hours later when compared with that of a non-etched sample. On the other hand, we were unable to confirm the effectiveness of the recovery. From these results, the recovery of damage caused by acid etching cannot be expected when a tooth is exposed to a calcifying solution. However, the finding demonstrated that the crystallized layer was apatite.
\end{abstract}

\author{
Key words \\ Apatite, \\ FT-Raman, \\ Remineralization, \\ Tooth enamel, \\ $\mathrm{X}$-ray diffraction
}

\section{Introduction}

Decalcified enamel surfaces have been studied both of clinically ${ }^{1-3)}$ and by scanning electron microscopy ${ }^{4,5}$. The reacted products formed on the surfaces of tooth enamel after remineralization have been examined by X-ray diffractometry and infrared spectroscopy ${ }^{6,7)}$. However, a definitive evaluation has not been made on the reacted product in comparison with undecalcified enamel, since the quantities of new mineral are extremely limited relative to the mass of the starting sample.

In order to carry out a more detailed examination of the reacted products on the enamel surface in this study, the reacted products were increased in quantity by using enamel powder samples in which the surface area of the sample became a maximum,

Received on February 20, 2006

Accepted on July 18, 2006 and these were analyzed by Fourier Transform Raman Spectrophotometry (FT-Raman) ${ }^{8)}$ and by $\mathrm{X}$-ray diffraction and by Thermogravimetry.

\section{Materials and Methods}

\section{Materials}

\section{Preparation of the extracted teeth}

Five maxillary central incisors, extracted by periodontosis and stored in distilled water, without macroscopic dental caries, morphological abnormality, or calcification abnormality on the labial surface were used. Polishing was carried out with emery abrasive paper \#400, after the labial surface was cleaned by a polishing brush.

\section{Preparation of enamel powder}

Human enamel powder (hereafter abbreviated as enamel powder) was prepared from 20 sound permanent teeth. The tooth surfaces were cleaned with a polishing brush. Then the dentin was ground off 


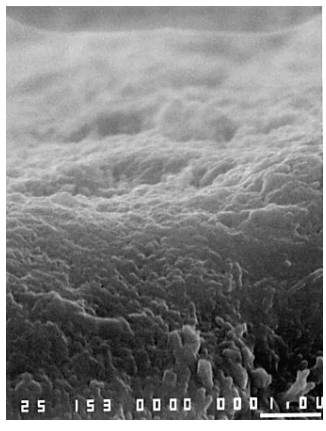

etching

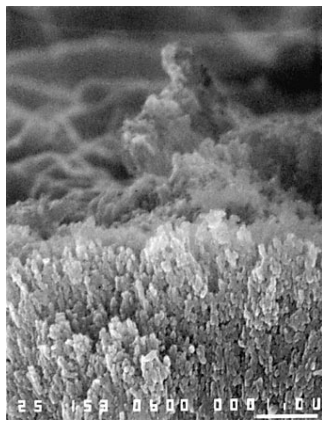

$6 \mathrm{~h}$

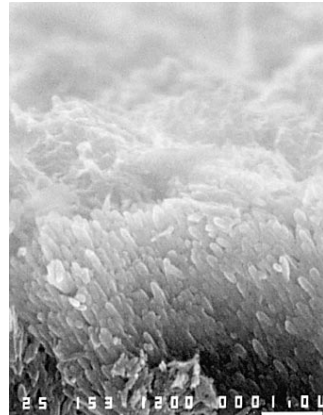

$12 \mathrm{~h}$

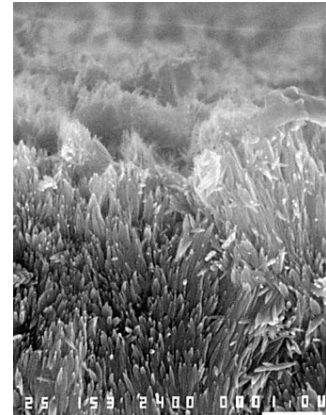

$24 \mathrm{~h}$

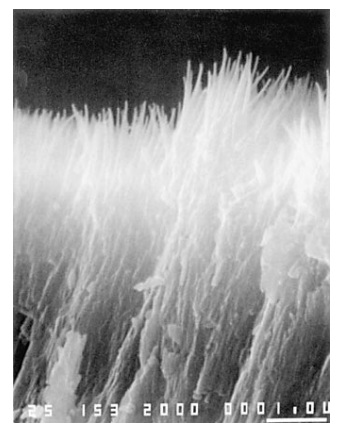

$48 \mathrm{~h}$

Fig. 1 SEM images of enamel surfaces of extracted tooth after calcification treatment

with a steel bur as much as possible and the enamel was crushed into fine particles with a crusher. Particles from 74 to $150 \mu \mathrm{m}$ (\#200 to 100) were connected using JIS standard filters to produce sample powders.

\section{Remineralization solution}

The remineralization solution (Ten Cate solution ${ }^{9)}$ of composition: $2 \mathrm{mM} \mathrm{Ca}, 1.2 \mathrm{mM}$ phosphate, $1 \mathrm{ppm} \mathrm{F}$, $\mathrm{pH}=7.1-7.2)$ was adopted and prepared immediately before use.

\section{Methods}

\section{Teeth}

The samples were decalcified using $10 \mathrm{ml}$ of $10 \%$ phosphoric acid for 20 seconds, and deionized water was used for cleaning and removal of the acid. The duration of immersion in the remineralization solution was set to $6,12,24$ or 48 hours and then each sample was divided by a stainless steel knife and hammer. SEM images of the samples were observed under an electron probe micro analyzer (EPMA) (JCMA-733, JEOL) (acceleration voltage: $25 \mathrm{kV}, 3,000$ times photographing magnification) immediately after decalcification and 48 hours after remineralization and compared with the sample before decalcification. For analysis of a cross section of a sample, the sample after 48 hours was embedded in epoxy resin. Gold evaporation was done and it was examined by $\mathrm{X}$-ray reflections $(\mathrm{CaK} \alpha, \mathrm{PK} \alpha)$ (acceleration voltage $20 \mathrm{kV}$, sample current $5 \mathrm{~mA}$, photographing magnification 4,000 times).

\section{Powder}

The enamel powders were dried before use and $100 \mathrm{mg}$ was weighed on a Millipore filter. The powder was decalcified using $10 \mathrm{ml}$ of $10 \%$ phosphoric acid for 20 seconds, and $500 \mathrm{ml}$ of deionized water was aspirated and poured onto the Millipore filter for cleaning and removal of the acid. Then the powder was dried in an oven with silica gel $\left(55^{\circ} \mathrm{C}\right)$ for 18 hours and $150 \mathrm{~m} l$ of a preheated remineralization solution was added to the decalcified powder. The solution was replenished occasionally to compensate for calcium loss. After static storage in an incubator at $37^{\circ} \mathrm{C}$ for a fixed time, the powder was dehydrated and dried in an oven with silica gel $\left(55^{\circ} \mathrm{C}\right)$ for 18 hours. The duration of immersion in the remineralization solution was set to $6,12,24$, or 48 hours. For comparison with the remineralization solution, deionized water was used as a control.

Measurements were conducted on five samples of the powder. In order to examine the weight variation by the decalcification and remineralization, 


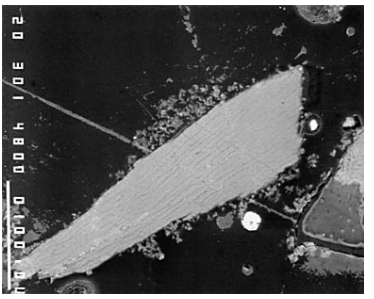

powder

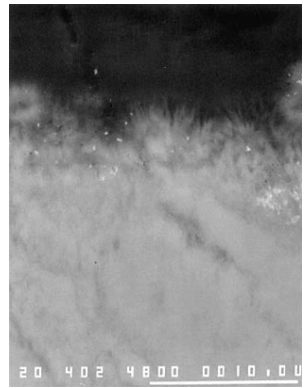

composition image

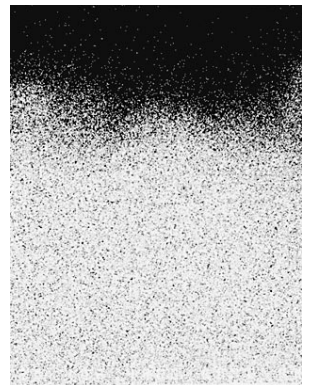

Ca K $\alpha$ characteristic $\mathrm{X}$-ray images

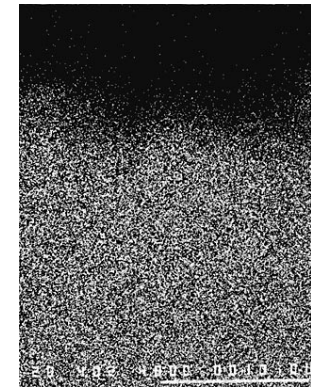

P K $\alpha$ characteristic $\mathrm{X}$-ray images

Fig. 2-1 Section analysis of the powder after remineralization

A section by composition image, X-ray image $(\mathrm{CaK} \alpha$ and $\mathrm{PK} \alpha)$ analyzed enamel powder for 48 hours using EPMA.

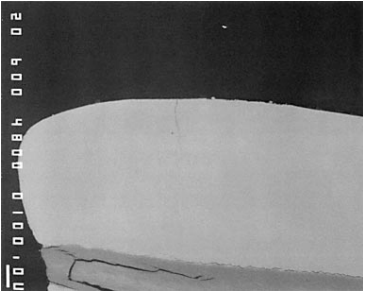

teeth

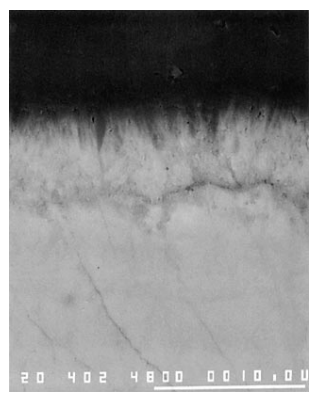

composition image

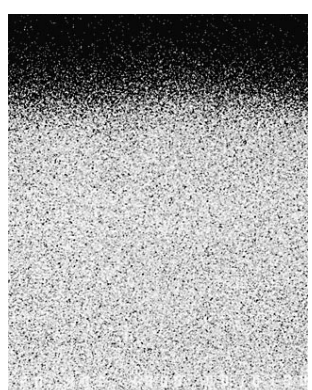

$\mathrm{Ca} \mathrm{K} \alpha$ characteristic X-ray images

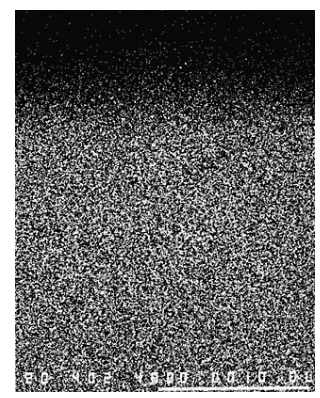

$\mathrm{P}$ K $\alpha$ characteristic $\mathrm{X}$-ray images

Fig. 2-2 Section analysis of the teeth after remineralization

A section by composition image, X-ray image $(\mathrm{CaK} \alpha$ and $\mathrm{PK} \alpha)$ analyzed teeth enamel for 48 hours using EPMA.

the samples were measured at specified times on a draft-shielded microbalance (AC120S, Sartorius) (temperature $20 \pm 2^{\circ} \mathrm{C}$, relative humidity $40 \pm 5 \%$ ). SEM images of the samples were observed by the same method as for the teeth. The products, 48 hours after immersion in calcifying solution, were studied using X-ray diffraction (RAD-r, Rigaku) (the X-ray source $\mathrm{CuK} \alpha$, tube voltage $50 \mathrm{kV}$, tube current 


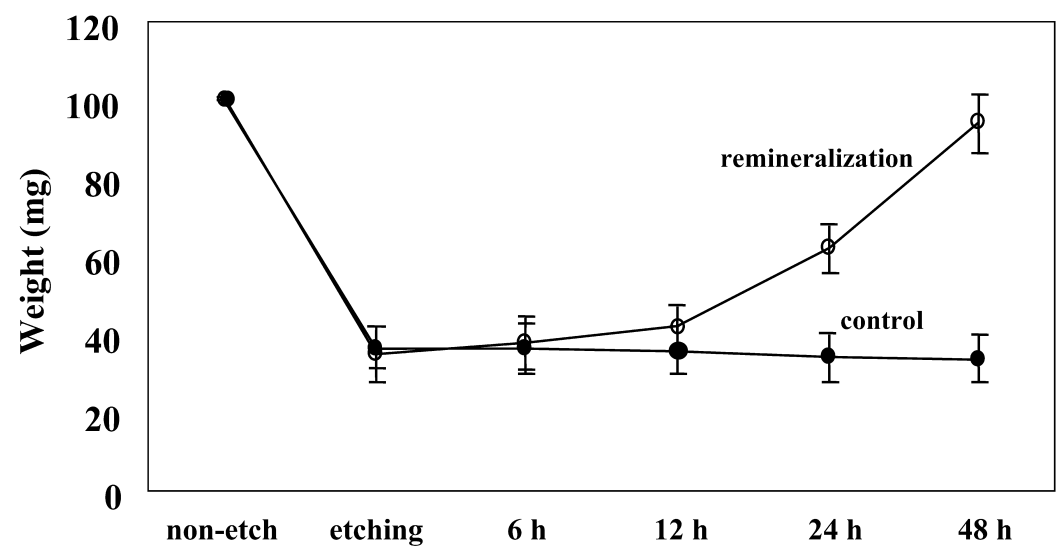

Fig. 3 Weight change of enamel powders

Remineralization group, the weight of enamel powders increased as the processing time increased. In the control group using distilled water, enamel powder showed a significant weight change after the start of the experiment.

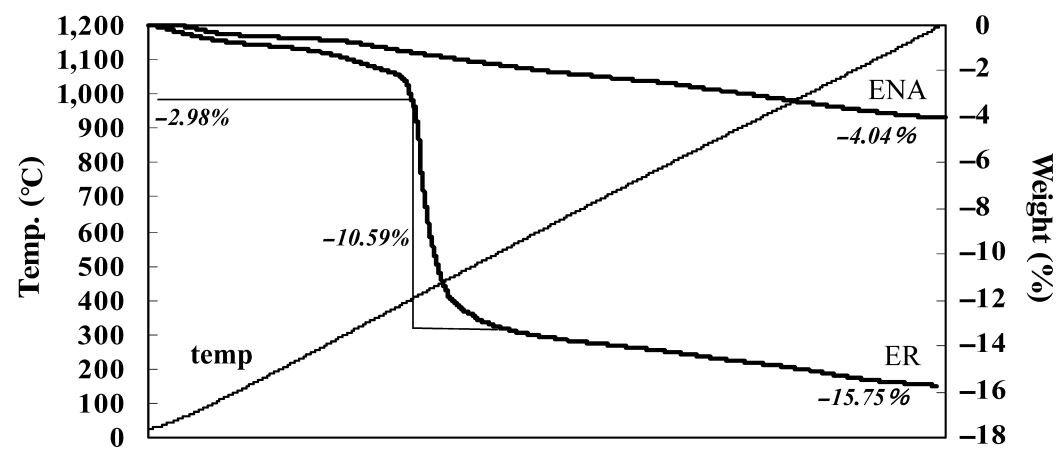

Fig. 4 Thermogravimetry (TG)

This summarized thermogravimetric changes in the enamel powders of non-etched (ENA) and 48 hours after immersion in the remineralization solution (ER). The powders showed clearly different thermogravimetric changes with increasing temperature immediately after the start of measurement.

$30 \mathrm{~mA}$, angular range $2 \theta=15-70$ degrees) for the purpose of comparison with the untreated sample. $\mathrm{X}$-ray reflections $(\mathrm{CaK} \alpha, \mathrm{PK} \alpha)$ of the sample after 48 hours was also examined by the same method as for the teeth. The products were then studied with a thermogravimetry differential thermal analyzer (TG8100, Rigaku), and by X-ray diffraction (RAD-r, Rigaku). They were also analyzed by FT-Raman spectroscopy (JRS-FT7000, JEOL) for the purpose of comparison with original enamel. The analysis conditions were as follows: measurement wavevector $250-3,800 \mathrm{~cm}^{-1}$, energy source a YAG laser (1,064 $\mu \mathrm{m}$ wavelength), beam spot 3 umq, output: $50 \mathrm{~mW}$ or $250 \mathrm{~mW}$.

The protocol of the study was approved by the research ethics committee of Meikai University.

\section{Results}

\section{Observation of the teeth}

Figure 1 shows the SEM images of a cross-section of tooth enamel. Needle like crystal growth was seen on the enamel substrate with progress in the time of calcification treatment.

Figures 2-1, 2-2 show a cross-sectional analysis of tooth enamel and powders which were immersed in calcifying solution for 48 hours. According to the composition image, the layer of product material of thickness about $4 \mu \mathrm{m}$ was confirmed on the etched enamel surface and powders. There was no differences for the distribution of $\mathrm{Ca}$ and $\mathrm{P}$ of this layer from the $\mathrm{CaK} \alpha$ and $\mathrm{PK} \alpha$ characteristic X-ray images. 


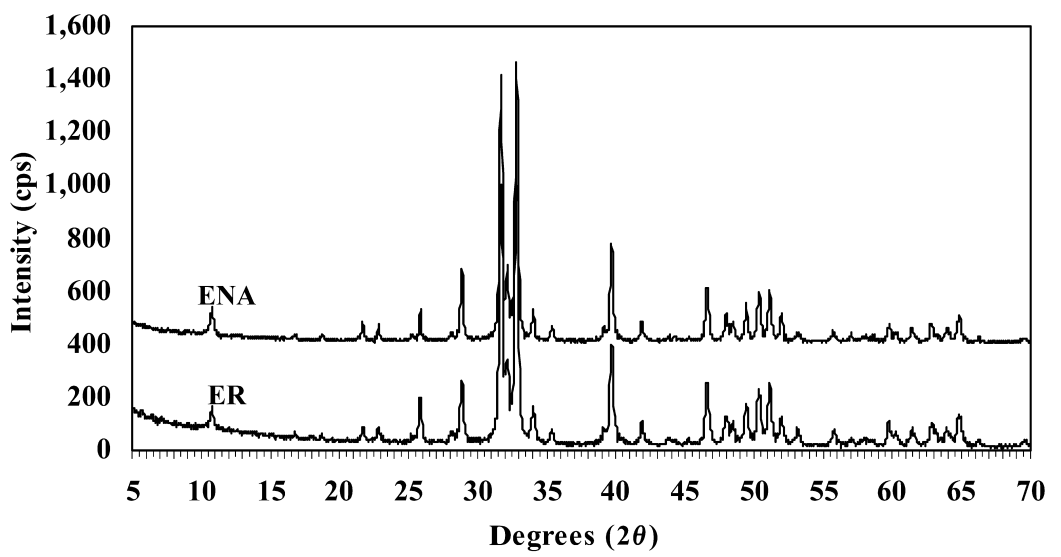

Fig. 5 X-ray diffraction

A spectrum of X-ray diffraction for remineralization (ER) was compared with non-etched (ENA) in enamel powder. $\left(5-70^{\circ}\right)$

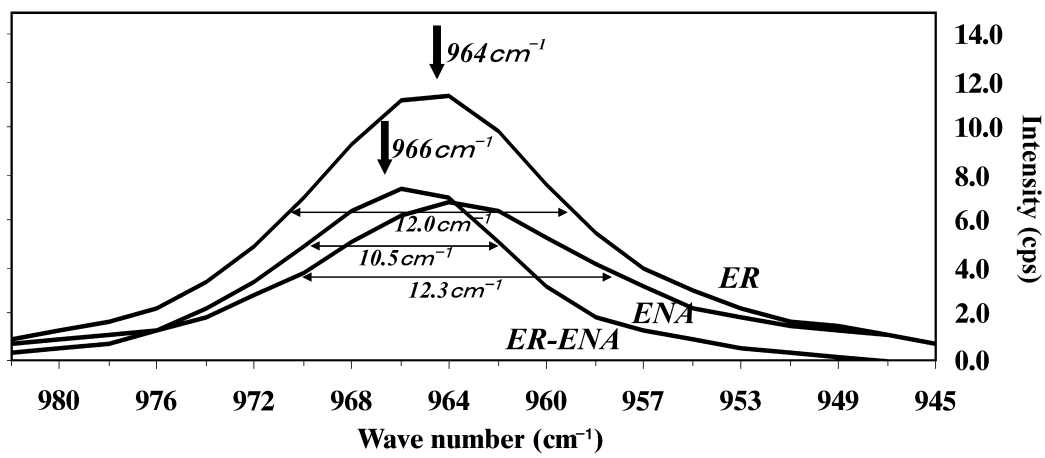

Fig. 6 PO stretching vibrations

When the enamel powder group was compared non-etched (ENA) and remineralization (ER), the spectral peak did not change and the width at half peak height remained. But a peak was recognized to peak position of apatite composition when a deduction, a wave pattern divided ENA from ER.

\section{Weight change of enamel powders}

The initial weight of the enamel powder was $100 \mathrm{mg}$, but it was decreased to $34.98 \pm 6.92 \mathrm{mg}$ after the decalcification. It then increased to $94.04 \pm 7.35 \mathrm{mg}$ 48 hours after immersion in calcifying solution, while the powder immersed in distilled water did not increase in weight after decalcification (Fig. 3). The weight change in each powder following each process was analyzed by a one-way ANOVA.

Thermogravimetry (TG)

Figure 4 summarized thermogravimetric changes in the enamel powders after remineralization. A decrease of about $4.04 \%$ was observed in the nonetched enamel powder (ENA) at $1,200^{\circ} \mathrm{C}$. Decreases of about $2.98 \%$ at $400^{\circ} \mathrm{C}$, about $13.57 \%$ at $600^{\circ} \mathrm{C}$ and about $15.75 \%$ at $1,200^{\circ} \mathrm{C}$ occurred in the etched powder after remineralization (ER). The powders showed clearly different thermogravimetric changes with increasing temperature immediately after the start of measurement.

\section{$\mathrm{X}$-ray diffraction}

Figure 5 showed X-ray diffraction patterns of the non-etched enamel powder (ENA) and the etched enamel powder 48 hours after immersion in calcifying solution (ER). Compared with ENA, products other than hydroxyapatite (ICDD card 09-0432), such as calcium fluoride (ICDD card 48-1298, 35-0816), were not recognized in ER.

\section{FT-Raman analysis}

Figure 6 summarized the FT-Raman spectra for the non-etched enamel powder (ENA) and the enamel powder 48 hours after immersion in calcifying 
solution (ER). The absorption spectra of the enamel powders with respect to $\mathrm{PO}$ stretching vibrations stemmed from $\mathrm{P}$ and $\mathrm{O}$ in the apatite.

There were no differences of the half-height widths and the position of the peak of the spectrum between ENA and ER. When the waveform was separated (ENA was deducted from ER), the peak of the spectrum was located at $966 \mathrm{~cm}^{-1}$ which is the value of the peak of synthetic apatite, and the half-height width was $10.5 \mathrm{~cm}^{-1}$.

\section{Discussion}

Remineralization is a phenomenon in which new crystals precipitate and are deposited inside enamel in which a subsurface lesion has been created by an acid. This is different from the phenomenon of crystal deposition onto the surface of decalcified enamel $^{10)}$. Numerous reports ${ }^{1-3,5-11)}$ have dealt with the recovery of an acid-processed enamel. The phenomena discussed in this report can be classified as belonging to the latter process. In the present in vitro experiment, crystals deposited onto the surface of decalcified enamel powders and the tendency for recovery of acid-treated enamel was verified.

In the group which was immersed in distilled water, there was no weight variation. However, in the group which was immersed in the remineralization solution, there was a weight increase, and the increment appeared, from the SEM images, to be due to deposition of needle like crystals. According to the TG, about $86.43 \%$ of the weight of the powder which increased during the remineralization was apatite. The X-ray diffraction also showed that the product material was based on the generation of apatite because there was no peak except for that of apatite in enamel powder after the 48 hours. In the FT-Raman analysis, PO stretching vibrations did not show any difference of the half-height widths and the position of the peak of the spectrums between ENA and ER. Therefore, the deposited crystals seem to be similar to the original apatite. For the enamel powder, the half-height width was extended but the entire spectral shape at 48 hours after remineralization (ER) shifted to the high frequency side. This may be attributable to an increase in apatite, because the spectrum from the enamel powder was similar to that of the apatite generated by remineralization.

In the experiment in which the extracted tooth was immersed in the remineralization solution, there were no difference in comparison with the experi- ment using the enamel powder on the composition image and the distribution of $\mathrm{Ca}$ and $\mathrm{P}$. In the SEM image of the extracted tooth, the crystals grew as needles with the time, and extended from the enamel surface in an axial direction.

It has been reported that the recovery after acid treatment in vivo on the enamel surface was due to deposition of organic material and of non-structured sediment in vitro ${ }^{5}$. However, the material formed in this experiment appears mainly to be apatite.

In this in vitro research, the weight increase was confirmed by the generation of apatite. However, from in vivo aspect, the formation of apatite crystals in a short period seems to be difficult because of the effects of the fluoride concentration in the saliva, organic materials in the saliva and $\mathrm{Ca} / \mathrm{P}$ ratios and of the repetition of re- and de-mineralization at the surface of tooth in the mouth.

In research on the recovery after acid treatment of the enamel using clinical observation of SEM and microradiography, many studies have been reported in which there was little injury to the enamel by the acid treatment ${ }^{4,12,13)}$. However, from present morphological observation and thermogravimetric analysis, the damage of the enamel received by the acid treatment was large and also decreased the crystallinity of the apatite. And perfect recovery of enamel could not recognized. From the present experiment, although it was not possible for the enamel crystals to recover perfectly after the acid treatment, it was shown that the deposited apatite seemed to have a possibility of fulfilling a role as a store of $\mathrm{Ca}$ and $\mathrm{P}$ which maintains chemical equilibrium of the dissolution by the acid. Since the deposit consisted of apatite, the results may be fully applicable to clinical cases in the future.

\section{Conclusions}

This study showed that most of the products which deposited during an exposure to a remineralization solution consisted of apatite after acid treatment of enamel.

\section{References}

1) Buonocore, M.G.: A simple method of increasing the adhesion of acrylic filling materials to enamel surfaces. J Dent Res 34: 849-853, 1955.

2) Retief, D.H., Dreyer, C.J. and Gavron, G.: The direct bonding of orthodontic attachments to teeth by means of an epoxy resin adhesive. Am J Orthod 58: 
21-40, 1970.

3) Arana, E.M.: Clinical observations of enamel after acid-etch procedure. JADA 89: 1102-1106, 1974.

4) Albert, M. and Grenoble, D.E.: An in vivo study of enamel remineralization after acid etching. $J S$ Calif Dent Ass 39: 747-751, 1971.

5) Garberoglio, R. and Cozzani, G.: In vivo effect of oral environment on etched enamel: A scanning electron microscopic study. J Dent Res 58: 1859$1865,1979$.

6) Kamiura, M.: Surface remineralization following the acid etching of human enamel. $J J$ Ped Dent 23: 600-625, 1985.

7) Takamizawa, Y.: A study on the ultrastructure of remineralized layers on the acid-etched enamel. Tsurumi Univ Dent J 19: 41-56, 1993.

8) Penel, G., Leroy, G., Rey, C. and Bres, E.: MicroRaman spectral study of the $\mathrm{PO}_{4}$ and $\mathrm{CO}_{3}$ vibrational modes in synthetic and biological apatites. Calcif Tissue Int 63: 475-481, 1998.

9) Ten Cate, J.M. and Arends, J.: Remineralization of artificial enamel lesions in vitro. Caries Res 11: 277286, 1977.

10) Suga, S.: Control of progress of enamel caries - Is it possible by remineralization?-. The Quintessence 4: 860-877, 1985.

11) Lenz, H. and Mühlemann, H.R.: In vivo and in vitro effects of saliva on etched or mechanically marked enamel after certain periods of time. Helv Odonto Acta 7: 30-33, 1963.

12) Silverstone, L.M.: Etching teeth with acid. Brit Dent $J$ 135: 95, 1973.

13) Silverstone, L.M.: Fissure sealants: The susceptibility to dissolution of acid-etched and subsequently abraded enamel in vitro. Caries Res 11: 46-51, 1977. 